\title{
A rapid sensitive, flow cytometry-based method for the detection of Plasmodium vivax-infected blood cells
}

\author{
Wanlapa Roobsoong ${ }^{1,2}$, Steven P Maher ${ }^{1}$, Nattawan Rachaphaew ${ }^{2}$, Samantha J Barnes ${ }^{1}$, Kim C Williamson ${ }^{3}$, \\ Jetsumon Sattabongkot ${ }^{2}$ and John $\mathrm{H}_{\text {Adams }}{ }^{*^{*}}$
}

\begin{abstract}
Background: Plasmodium vivax preferentially infects Duffy-positive reticulocytes and infections typically have few parasite-infected cells in the peripheral circulation. These features complicate detection and quantification by flow cytometry (FC) using standard nucleic acid-based staining methods. A simple antibody-based FC method was developed for rapid parasite detection along with simultaneous detection of other parasite and erythrocyte markers.

Methods: Clinical samples were collected from patients diagnosed with P. vivax at a district Malaria Clinic in Kanchanaburi, Thailand. One $\mu \mathrm{L}$ of infected blood was washed, fixed, stained with a Plasmodium pan-specific anti-PfBiP antibody conjugated with Alexa Fluor 660, and analysed by FC. Additional primary conjugated antibodies for stage-specific markers of $P$. vivax for late trophozoite-early schizonts (MSP1-Alexa Fluor 660), late-stage schizonts (DBP-Alexa Fluor 555), and sexual stages (Pvs16) were used to differentiate intra-erythrocytic developmental stages.
\end{abstract}

Results: The percentages of $P$. vivax-infected cells determined by the FC method and manually by microscopic examination of Giemsa-stained thick blood smears were positively correlated by Spearman's rank correlation coefficient $\left(R^{2}=0.93843\right)$ from 0.001 to $1.00 \%$. vivax-infected reticulocytes.

Conclusions: The FC-based method is a simple, robust, and efficient method for detecting $P$. vivax-infected reticulocytes.

Keywords: Malaria, Vivax malaria, Plasmodium vivax, Flow cytometry, Diagnosis

\section{Background}

Diagnosis of malaria parasites in field isolates typically relies on light microscopic detection of Plasmodiuminfected blood cells in Giemsa-stained smears. Infection levels are often low and accurate determination of a parasitaemia requires the use of thick Giemsa-stained blood smears instead of thin blood smears used in acute infections or for monitoring in vitro cultures. Direct light microscopic observation Plasmodium-infected reticulocytes in thick smears is a slow procedure that is reliant on a skilled microscopist to manually count infected reticulocytes, since the parasites' morphologic features and staining patterns are distorted in thick smears. The

\footnotetext{
* Correspondence: jadams3@health.usf.edu

${ }^{1}$ Department of Global Health, College of Public Health, University of South Florida, Tampa, FL, USA

Full list of author information is available at the end of the article
}

difficulty of accurate identification and quantification is exacerbated with Plasmodium vivax, which is a leading cause of malaria in many countries [1], because parasitaemias are relatively low and the variable properties of the host reticulocytes [2,3].

In recent years numerous flow cytometric-based methods have been developed to detect and quantify Plasmodium falciparum in the laboratory [4-9]. Many of these methods use nucleic acid staining to detect parasite-infected cells, since nucleated white blood cells (WBCs) are removed for culture and mature erythrocytes retain virtually no nucleic acid. Gradually these methods are being adapted for use in field laboratories and clinics. However, blood samples directly from malaria patients frequently have many contaminating WBCs and anaemia in chronic infections can enhance reticulocytaemia. Therefore, analysis of clinical isolates and the preference of $P$. vivax for reticulocytes, 
which still retain abundant amounts of nucleic acids in the cytosol, complicates the use of the nucleic acid stain-based flow cytometry (FC) methods. This has led to investigation of alternative staining strategies to identify the parasiteinfected blood cells from field isolates. The objective of the study was to develop a strategy for rapid antibodybased staining to identify $P$. vivax-infected red blood cells as well as determine $P$. vivax stages of development. To identify parasite-infected blood cells, antibody to a Cterminal peptide epitope of PfBiP was used, which is a conserved cytoplasmic protein involved in endoplasmic reticulum (ER) retrograde trafficking [10,11]. Also known as the $78-\mathrm{kDa}$ glucose regulated protein and heat shock protein $70-2$, it is highly conserved amongst eukaryotic organisms [12]. Constitutive expression of $\mathrm{BiP}$ through asexual blood-stage development coinciding with continued ER development has led to the common use of anti-PfBiP as reference antigen in P. falciparum studies $[13,14]$. The antibodies to this conserved ER-resident protein were reactive with $P$. vivax and quantification of parasite-infected reticulocytes correlated accurately with light microscopic calculations.

\section{Methods}

\section{Fresh isolates human malaria parasites}

The Ethical Review Committee of Faculty of Tropical Medicine, Mahidol University, approved a Human Subjects protocol for this study. Fresh isolates of $P$. vivax were collected from symptomatic patients attending the malaria clinics in Kanchanaburi Province in districts near the western border of Thailand. After informed consent was obtained, a $20 \mathrm{ml}$ sample of $P$. vivax-infected blood was drawn by venipuncture into a $50 \mathrm{ml}$ tube containing heparin. Thick and thin smears were made from $1 \mu \mathrm{l}$ of packed blood cells before and after removal of WBCs. After being completely dried, thin blood film was fixed with absolute methanol for $30 \mathrm{sec}$. Both thick and thin blood films were stained with Giemsa at 1:10 dilution for $15 \mathrm{~min}$, rinsed with water, allowed to dry completely, and the sample was blinded when counting by light microscopy.

\section{Short-term in vitro culture of Plasmodium vivax}

WBCs were removed from $P$. vivax-infected blood by passage through Plasmodipur filter (EuroProxima) [15]. For short-term culture, filtered $P$. vivax-infected blood was incubated for 20-24 hr with McCoy's 5A medium (Sigma) supplemented with $25 \%$ heat inactivated human $A B$ serum (Interstate Blood Bank) in $T 75 \mathrm{~cm}^{2}$ tissue culture flask. The culture flask was placed in a sealed container purged under mixed gas 90:5:5\% N:O: $\mathrm{CO}_{2}$ and maintained at $37^{\circ} \mathrm{C}$ [16].

\section{Indirect immunofluorescence assay (IFA) Preparing IFA microscope slides}

Field isolates of $P$. vivax and a laboratory line of $P$. falciparum strain NF54 were used to prepare thin blood smears on microscope slides for IFA. Briefly, after 2024-hr culture P. vivax-infected blood was separated by $47 \%$ Percoll (Sigma) gradient centrifugation and an enriched fraction of schizont-stage parasites were collected at the gradient interface [16]. After three washes with PBS the enriched parasite fraction was diluted to $1 \%(\mathrm{v} / \mathrm{v})$ with PBS and $1 \mu \mathrm{l}$ of diluted parasite was spotted on multi-well slides. Plasmodium falciparum NF54 IFA microscope slides were prepared from cultures (RPMI1640 medium supplemented with 5\% Albumax to a 10\% parasitaemia) concentrated by centrifugation, washed extensively with PBS, and suspended to $1 \%(\mathrm{v} / \mathrm{v})$ with PBS. One $\mu \mathrm{l}$ aliquot of diluted parasite-infected reticulocyte suspension was spotted on multi-well slides, air dried at room temperature (RT), hermetically sealed, and stored at $-20^{\circ} \mathrm{C}$ until used.

\section{IFA staining}

Parasites were fixed with $4 \%$ paraformaldehyde for $20 \mathrm{~min}$ at RT, treated with $1 \%$ Triton X-100 (TX-100) for $20 \mathrm{~min}$ at RT, and washed three times with PBS containing Tween-20 (PBST). To minimize non-specific binding, IFA slides were treated with $3 \%$ BSA for $30 \mathrm{~min}$ at $37^{\circ} \mathrm{C}$ and washed 3 times with PBST. Finally, parasites were stained for $30 \mathrm{~min}$ at $\mathrm{RT}$ in a dark moist chamber with anti-PfBiP (MRA28) Alexa Fluor 660 at a 1:100 dilution alone and in combination with anti-DBP (3D10) Alexa Fluor 488 at 1:100 dilution or with anti-PvMSP119 Alexa Fluor555 at 1:100 dilution. For staining of gametocytes, parasites were stained with anti-pvs16 for 30 min at RT. After washing with PBST a combination of anti-PfBiP Alexa Fluor 660 at 1:100 diluion and Goat anti-mouse Alexa Fluor 488 at 1:500 dilution was added. After staining, microscope slides were washed three times, DAPI plus anti-fade was applied to the slide, and the slide was covered with a cover slip. Parasites were observed by epifluorescence and phase contrast microscopy (Olympus IX71 and DeltaVision CORE) and confocal microscopy (Zeiss LSM700). Micrograph images were prepared for publication using SoftWorx (Applied Precision) and ZEN (Zeiss).

\section{Direct conjugation of antibodies}

Total IgG was purified from anti-PfBiP [11] rabbit sera (MRA28) and anti-PvMSP1-19 (MRA30) rabbit sera using a Protein G column (HiTrap Protein G HP, GE Healthcare). One milligram of purified IgG from each antibody was directly conjugated with Alexa Fluor fluorescence dye (Molecular Probe) according to manufacturer protocol. Anti-PfBiP purified rabbit IgG was conjugated with Alexa 
Fluor 660, anti-PvMSP1-19 purified rabbit IgG was conjugated with Alexa Fluor 555, and anti-DBP (3D10) monoclonal antibody was conjugated with Alexa Fluor 488. All direct conjugated antibodies were aliquot and kept at $-20^{\circ} \mathrm{C}$.

\section{Sample preparation for flow cytometry}

One $\mu \mathrm{L}$ of infected blood was suspended in $100 \mu \mathrm{L}$ of wash buffer PBS-B (PBS + 0.05\% BSA) and fixed with $100 \mu \mathrm{L}$ of $0.05 \%$ glutaraldehyde for $5 \mathrm{~min}$ at RT. Post fixation, cells were washed in PBS-B, treated with $100 \mu \mathrm{L}$ of $0.3 \%$ TX-100 for 5 min at RT, washed twice in PBS-B, and blocked with 3\% BSA for $5 \mathrm{~min}$ at RT. After washing in PBS-B, samples were processed for antibody staining at RT for $10 \mathrm{~min}$ in the dark, using $100 \mu \mathrm{L}$ of the following: (i) anti-PfBiP Alexa Fluor 660 (1:100); (ii) anti-PvMSP1-19 Alexa Fluor 555 (1:100) (iii) anti-DBP (3D10) [17] Alexa Fluor 488; and, (iv) anti-Pvs16 (1:100) (mouse antiserum prepared against bacterial-expressed product) followed by secondary antibody staining (washed $1 \mathrm{X}$ PBS-B and then incubated with $100 \mu \mathrm{L}$ of goat anti-Mouse IgG FITC $(1: 1,000)(\mathrm{DAKO}))$ for $10 \mathrm{~min}$ at RT in the dark). After washing with $100 \mu \mathrm{L}$ of PBS $+0.05 \%$ BSA, the sample was suspended with $400 \mu \mathrm{L}$ of PBS-B. Samples (ii), (iii) and (iv) were co-stained with anti-PfBiP Alexa Fluor 660 (1:100). As a control, $1 \mu$ l of packed uninfected blood, without removal of WBCs, was stained along with the infected blood and used as the control. The samples were kept in the dark at $4^{\circ} \mathrm{C}$ until FC analysis.

\section{Flow cytometry}

The analysis by FC was performed on an Accuri C6 (BD Biosciences) with standard optic configuration (488 nm blue laser and $633 \mathrm{~nm}$ red laser). The threshold was set at 80,000 on forward scatter (FSC-A). The sample was transferred to $12 * 75 \mathrm{~mm}$ round bottom tube. One million events were acquired for each sample. Data analysis was performed with CFlow Sampler version 1.0.227.4 (BD Biosciences).

\section{Statistical analysis}

A Spearman correlation was performed, using SAS 9.2 (Cary, NC, USA released 2008), to determine if there was a correlation between the parasitaemia calculated by microscopy and the parasitaemia calculated by flow cytometry.

\section{Results}

\section{Plasmodium BiP is conserved}

Plasmodium falciparum BiP is an abundantly expressed protein of the ER involved in retrograde transport. Previous studies identified PfBiP as a useful localization marker and as a reference standard loading control for analysis of relative levels of parasite protein expression [11,18,19].
The IFA results in this study reconfirmed these observations demonstrating this anti-PfBiP reacted with all intra-erythrocytic developmental stages of P. falciparum (Figure 1A). Localization patterns reflected temporal and spatial changes in localization consistent [with] morphology of the ER during asexual stage growth and development. The fluorescence signal was generally dispersed in the cytoplasm of ring-stage parasites, increasing in abundance during trophozoite and early schizont stages before concentrating in the perinuclear apical end of forming merozoites in late segmenting schizonts.

A comparison of the P. falciparum BiP peptide sequence (SGDEDVDSDEL of PF3D7_0917900) that was used to produce the anti-PfBiP antibodies to the orthologous $P$. vivax sequence (SADEDVDSDEL of PVX_099315) indicates that this sequence is highly conserved (Table 1). To investigate usefulness of anti-PfBiP as a marker for $P$. vivax, this antibody was tested for IFA reactivity to intra-erythrocytic developmental stages of $P$. vivax. The fluorescence signal was similar to the dispersed patterns in the cytoplasm of ring, trophozoite and early schizont when it is concentrated at the apical end of daughter merozoites in segmented schizont of $P$. vivax (Figure 1B). The anti-PfBiP was specific for the cytoplasm of the $P$. vivax and did not react to uninfected reticulocytes.

Stage-specific antibodies anti-PvMSP1 and anti-DBP (3D10) were evaluated in combination with anti-PfBiP to distinguish $P$. vivax developmental stages. In trophozoite/ early schizont stages, the anti-PvMSP1 showed the circular fluorescence pattern in close proximity to the parasite plasma membrane while anti-PfBiP showed the diffuse fluorescence pattern in the cytoplasm of the parasite (Figure 2). In late-stage segmented schizonts, anti$\mathrm{PfBiP}$ showed fluorescence signal at the apical end in close proximity to the nucleus of the merozoite while the antiPvMSP1 fluorescence pattern localized at the membrane surrounding the daughter merozoites. The anti-DBP (3D10) fluorescence was detected only in late schizonts and localized at the apical end immediately adjacent to BiP localization (Figure 2C). In P. vivax gametocytes, the anti-Pvs16 was widely distributed in the peripheral cytoplasm while anti-BiP staining was relatively less in a dispersed punctate pattern (Figure 2D).

\section{Flow cytometry gating parameters}

The rapid antibody IFA staining method using antiPfBiP Alexa Fluor 660 was adapted to blood cells in suspension and evaluated by FC for specificity in detecting $P$. vivax-infected reticulocytes. In this analysis, Thai adult uninfected blood was stained with anti-PfBiP Alexa Fluor 660 for use as a negative control to define cell populations with non-specific or background reactivity (Figure 3). In the uninfected sample, events were gated based on the FSC-A/SSC-A profile in the dot plot (P1). 


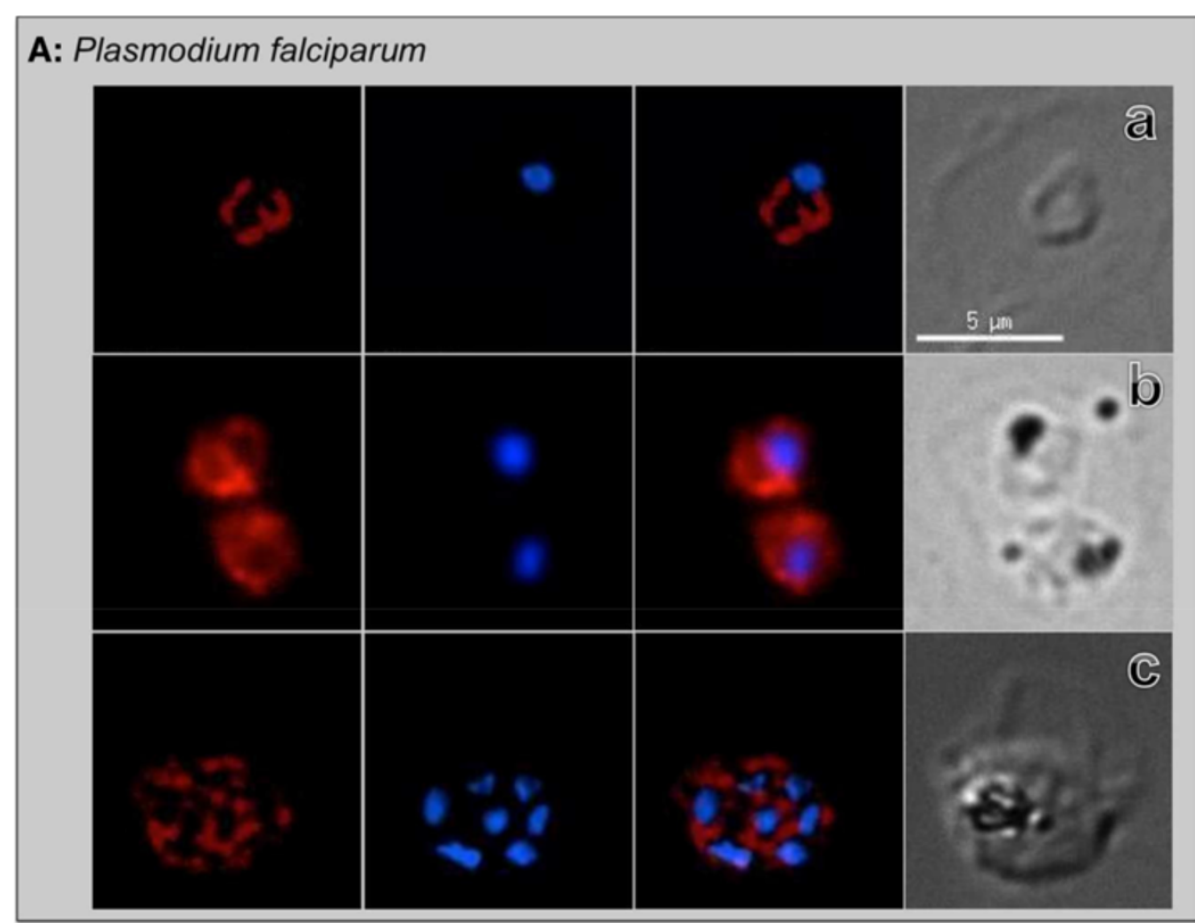

\section{B: Plasmodium vivax}

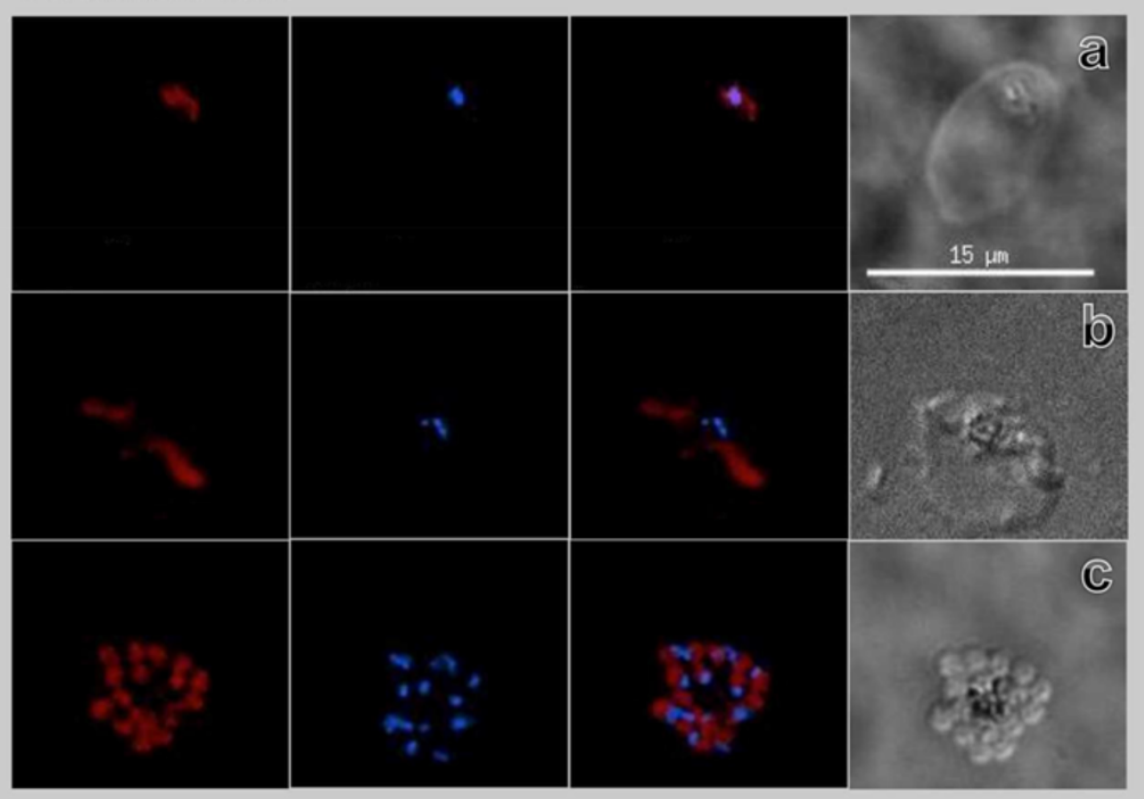

Figure 1 Reactivity of anti-PfBiP Alexa Fluor 660 to (A) Plasmodium falciparum ring, trophozoite and schizont stage and (B) Plasmodium vivax blood-stage parasites. Reactivity to ring, trophozoite and early schizont stage had a dispersed cytoplasmic fluorescence pattern (a-b) and in late-segmented schizonts was concentrated at the apical end of daughter merozoites (c) of both species.

The events in gate $\mathrm{P} 1$ were viewed in FSC-A/FSC-H profile to observe cell duplet. The single cells from gate P2 were selected to view in the anti-BiP Alexa 660-A/ FL2-A dot plot. The events in gate P3 were selected and viewed in anti-BiP Alexa 660-A/ FL2-A dot plot. In this plot, the events in gate P5 of the uninfected sample were considered as the background and were excluded from R1. In $P$. vivax-infected samples, parasites were identified as anti-BiP + events in gate R1 subtracted by the background in gate P5 (Figure 3). For identification of P. vivax late stages and gametocytes, the events were gated with the same strategy as for anti-BiP+, except the 
Table 1 Peptide sequences of BiP among Plasmodium species

\begin{tabular}{lll}
\hline Plasmodium species & Gene ID & Amino acid sequence \\
\hline P. falciparum & PF3D7_0917900 & SGDEDVDSDEL \\
P. vivax & PVX_099315 & SaDEDVESDEL \\
P. knowlesi & PKH_071520 & SGDEDVeSDEL \\
P. yoelii & PY05001 & pGDEDVDSDEL \\
& Consensus & ${ }^{* * D E D V * S D E L ~}$ \\
\hline
\end{tabular}

BiP peptide sequences from $P$. falciparum, $P$. vivax, $P$. knowlesi and $P$. yoelii were compared. The concensus peptide sequence of BiP (**DEDV*SDEL) was identified from $P$. falciprum BiP orthologs. Sequences were obtained from PlasmoDB (version 10.0) [20].

events were viewed in anti-MSP1 Alexa Fluor 555-A/ FL2-A (Figure 4), anti-DBP Alexa Fluor 488-A/ FL2-A (Figure 5) and anti-Pvs16-A/ FL2-A dot plot (Figure 6) for anti-PvMSP1-19, anti-DBP (3D10) and Pvs16 staining, respectively.

\section{Determination of parasitaemia and staging of the parasite}

The FC parameters for anti-PfBiP Alexa Fluor 660 detection was used to estimate the total number of $P$. vivax-infected reticulocytes in all samples. In 18 clinical samples, parasitaemias were determined both by anti-PfBiP FC and by direct light microscopic observation of Giemsa-stained thick blood smears. There was a significant correlation of the parasitaemias determined by FC and microscopy $\left(\mathrm{R}^{2}=0.94, \mathrm{P}<0.001, \mathrm{n}=18\right)$ (Figure 7A). To differentiate asexual developmental stages, anti-PfBiP was combined with stage-specific antibodies, anti-PvMSP1-19 and anti-DBP. MSP1-positive events identified stages beginning with mid-trophozoite/early schizonts through to completion of schizont development (MSP+). DBPpositive events were identified as beginning mid-schizont through to late-stage segmented schizonts (DBP+). Parasite stages positive for Pvs16 were identified as gametocytes (Pvs16+). Differential staining patterns were used to define the stages of development within each sample (Table 2). The number of ring and trophozoite stages was obtained by subtracting the number of $\mathrm{BiP}+$ events by the number of MSP1+ and Pvs16 + events while the number of early schizonts was obtained by subtracting the number of MSP1+ events by the number of DBP + events. The number of segmented schizonts and gametocytes were obtained directly from DBP + and Pvs16+ events, respectively. The parasitaemia of the individual asexual blood stages and the gametocyte-infected reticulocytes obtained from FC were compared with the corresponding values obtained from microscopy (Figure 7). There was a positive correlation of the ring stage parasitaemia (Figure 7B), schizont stage parasitaemia (Figure 7C) and gametocytaemia (Figure 7D) determined by FC and microscopy $\left(\mathrm{R}^{2}=0.8847(\mathrm{~N}=8), 0.6628(\mathrm{~N}=8)\right.$ and $0.5293(\mathrm{~N}=8)$, respectively).

To monitor the maturation of the parasites by flow cytometry, aliquots of $P$. vivax-infected blood was collected from short-term cultures at 12-hr and 24-hr intervals. The positive events from each stage-specific antibody were translated to maturation stage of the parasite (Table 2), using the strategy described above, and compared with those obtained from microscopic examination. There was an increase of MSP1+ and DBP + events while the number

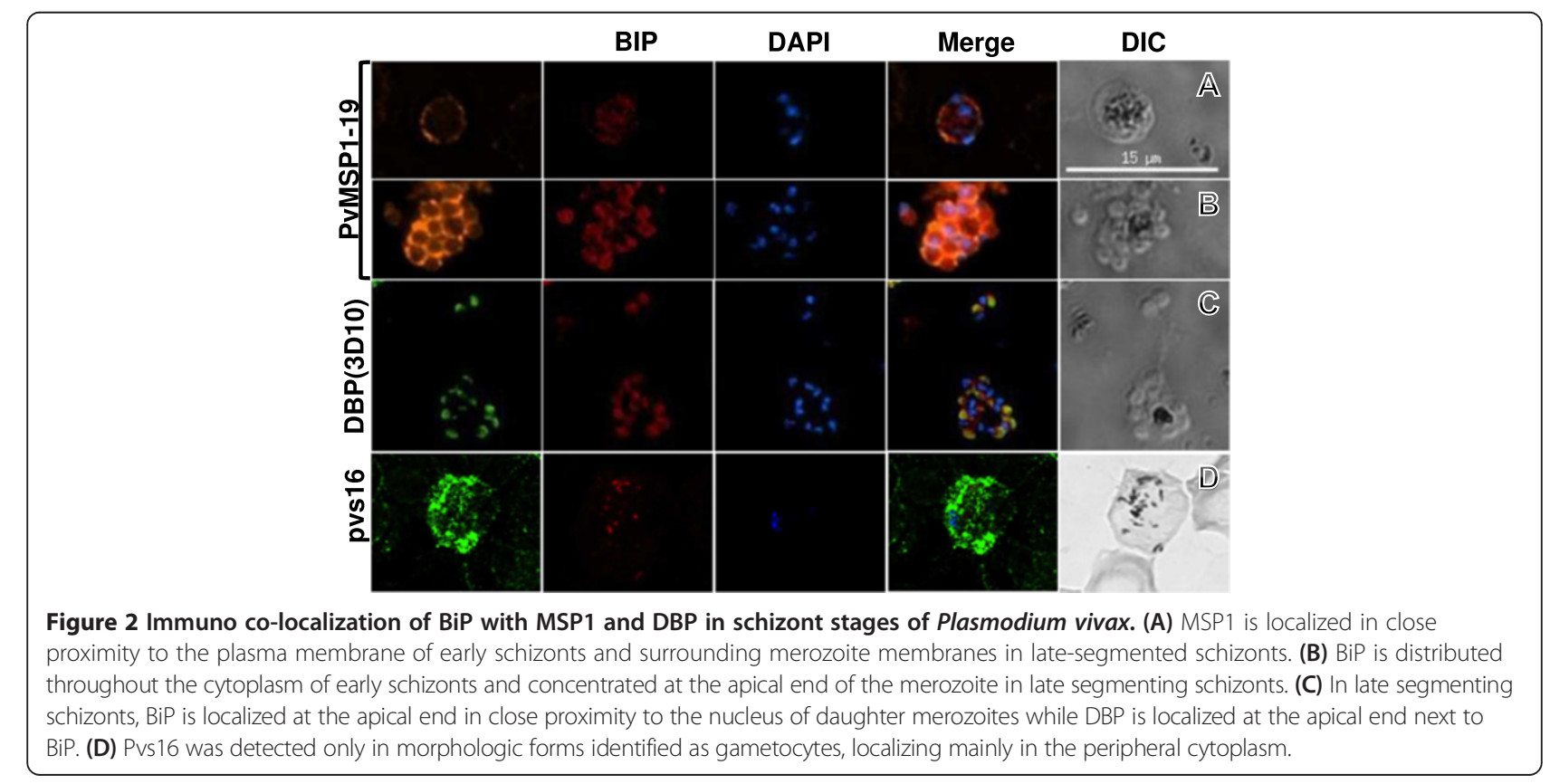




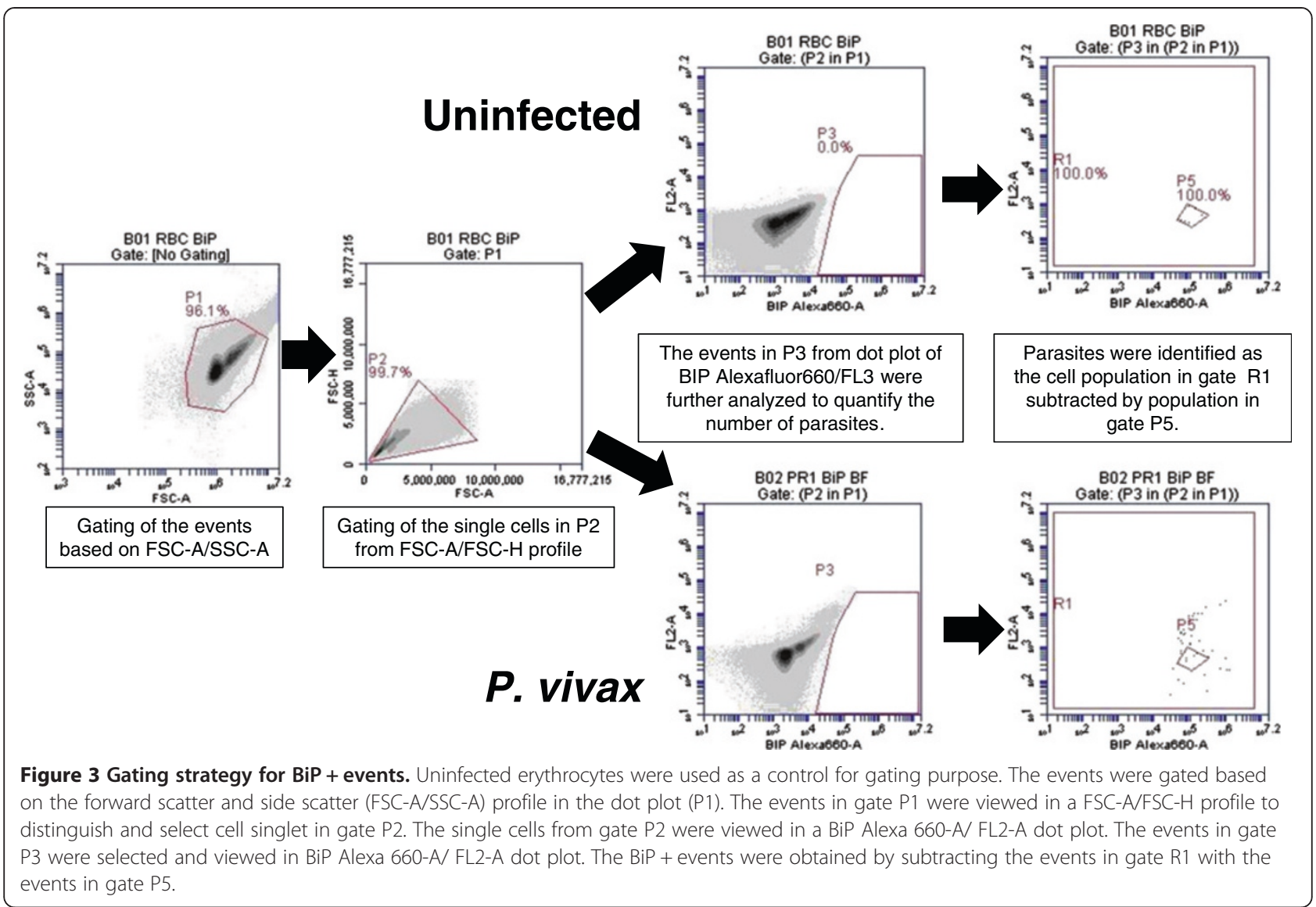

of $\mathrm{BiP}+$ events was similar during the examination period as determined by FC (Figure 8). Similar results were obtained by microscopic examination.

\section{Discussion}

Plasmodium falciparum $\mathrm{BiP}$ is defined as an endoplasmic reticulum resident protein involved in retrograde transport. Important for this study, the peptide sequence of PfBiP used to prepare an anti-PfBiP peptide serum is very similar among Plasmodium orthologues. Previous studies have demonstrated that $\mathrm{BiP}$ is constitutively expressed during asexual blood-stage development and the sera reacted specifically to the ER compartment of P. falciparum, Plasmodium berghei and Plasmodium yoelii $[11,21,22]$. In this study, the broad pan-species reactivity of the anti-PfBiP was used to quantify P. vivax blood stages in clinical isolates and short-term in vitro cultures, demonstrating that the anti-PfBiP can be used as a universal antibody to detect developing blood stages of both $P$. falciparum and $P$. vivax.

Counting the parasitaemia by light microscopy is still the gold standard, but it is a difficult, time consuming method requiring a high degree of training to accurately identify $P$. vivax in thick Giemsa-stained blood smears.
Flow cytometry is becoming a more accessible tool for many malaria research laboratories as the equipment becomes less expensive, more reliable and easier to use. Greater accessibility to FC has translated into more applications developed for P. falciparum research [23-25]. Most of the established methods for P. falciparum are based at least partly on nucleic acid staining. While these methods may be very useful for $P$. falciparum studies, they have been less valuable for $P$. vivax due to its preferential infection of reticulocytes that contain high levels of nucleic acids. The problem is compounded for analysis of in vitro and ex vivo cultures of $P$. vivax when reticulocytes derived from in vitro differentiation of hematopoietic stem cells are used and are contaminated with nucleated erythroid precursor cells.

\section{Conclusion}

The antibody-based staining that has been developed in this study offers the opportunity for a robust, simple method for sensitive real-time detection of the $P$. vivaxinfected cells in a standardized procedure that should reduce technical variation among researchers and laboratories. The reactivity of anti-PfBiP to both $P$. falciparum and $P$. vivax and any stages of blood-stage parasite offers 


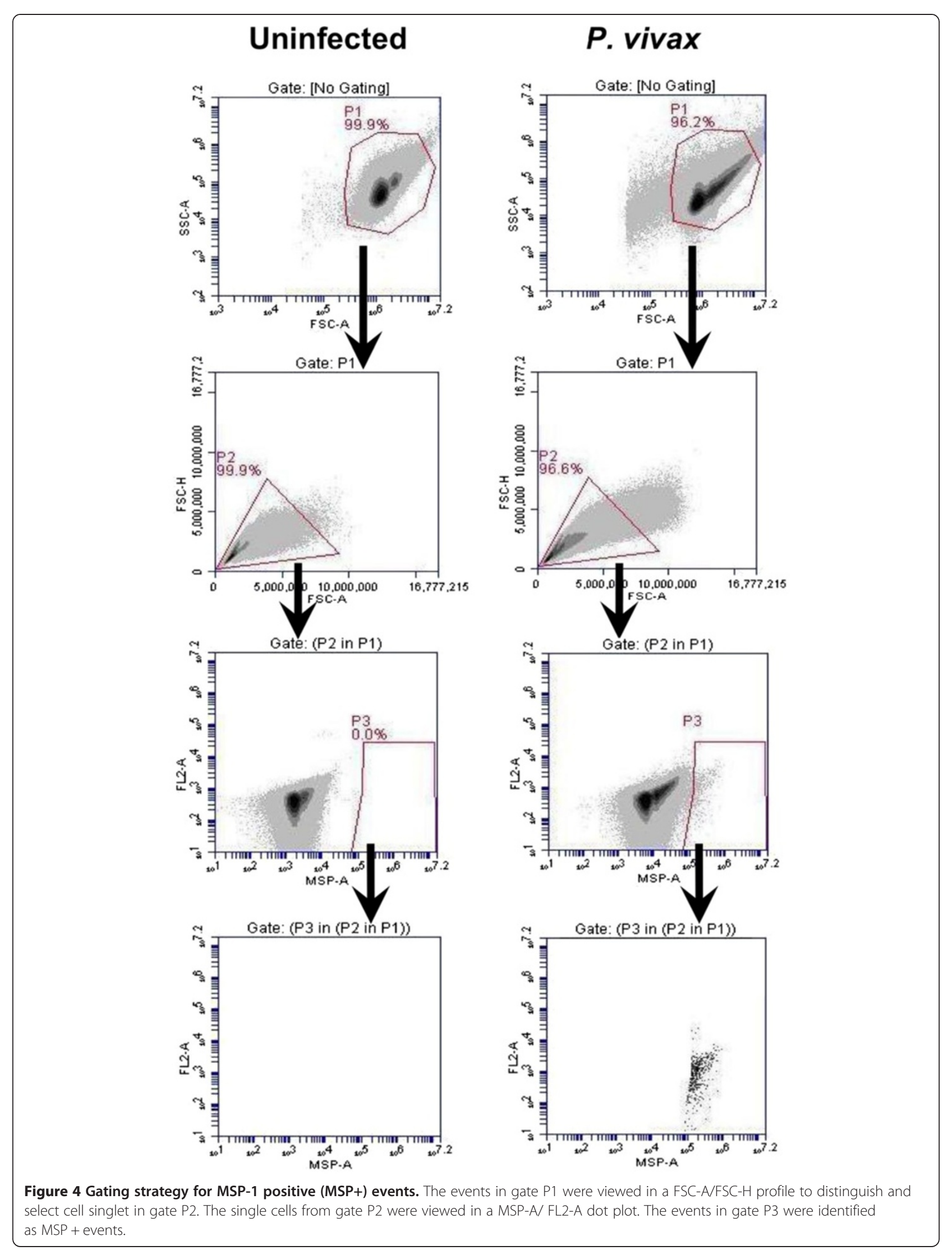




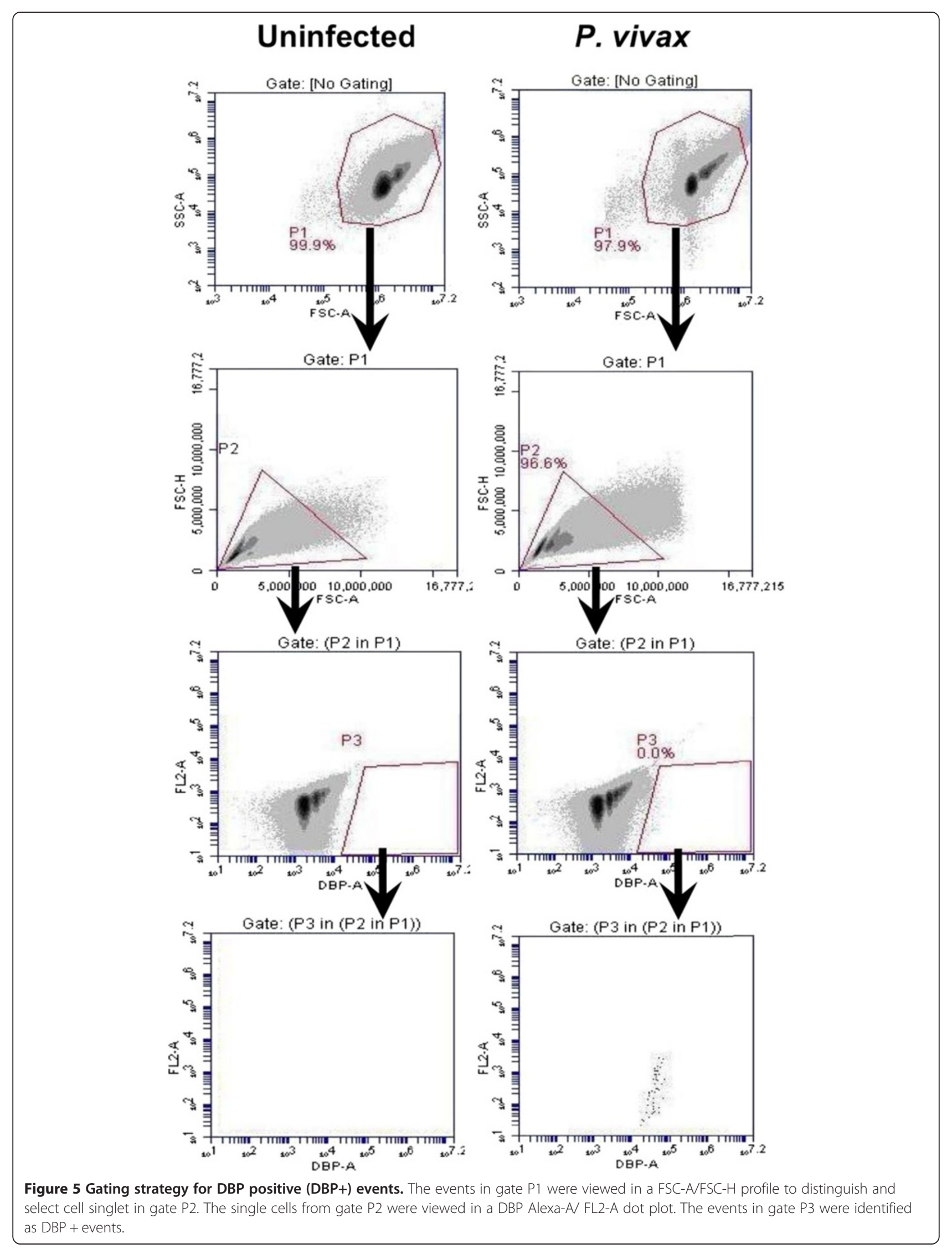




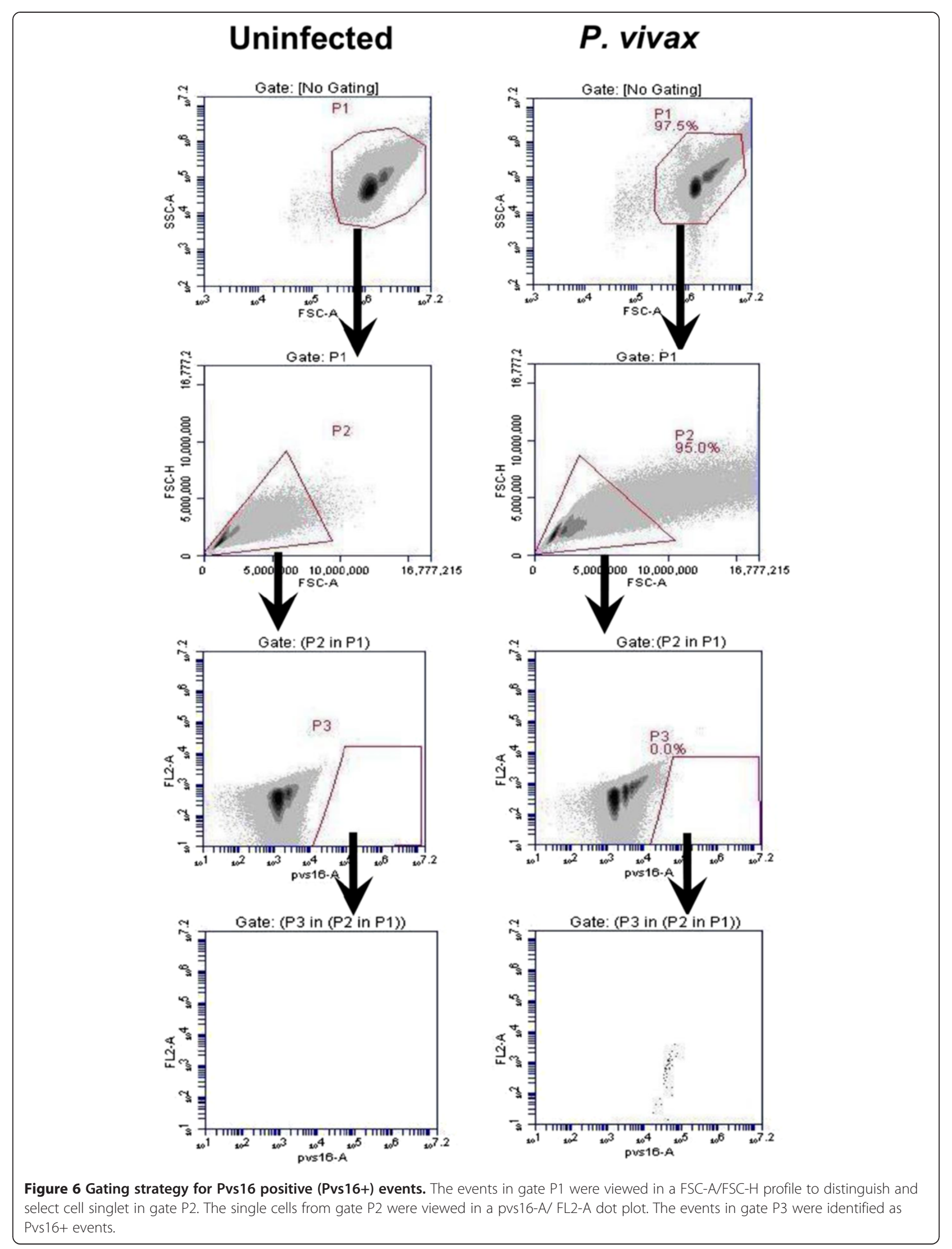




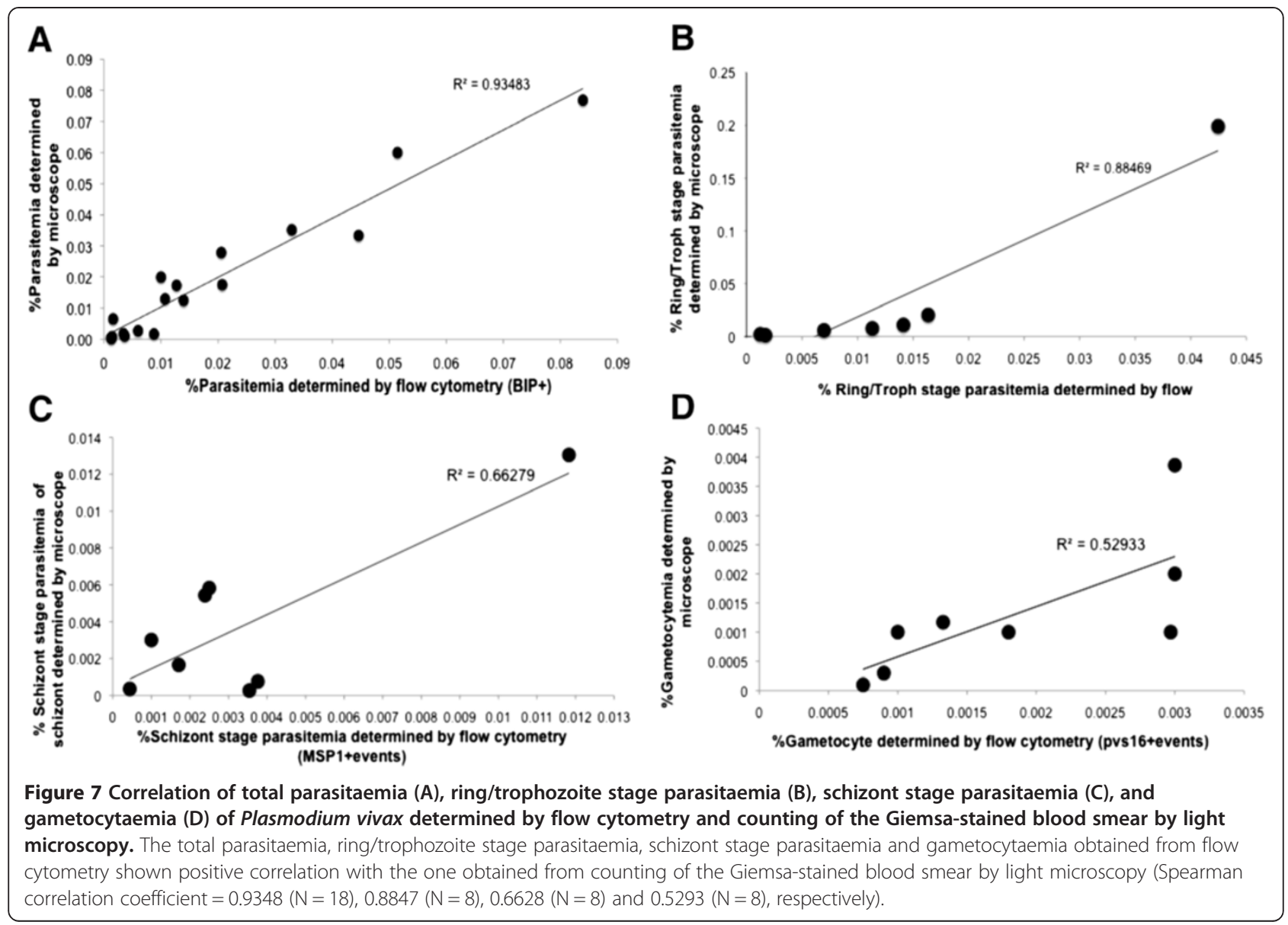

an advantage of using this antibody for determining the parasitaemia by flow cytometry. With an optimized protocol, the antibody-based FC can detect one parasite per one million cells count (ten parasites/1 $\mu$ l PRBC) and generates almost no background. When combined the anti-PfBiP with other stage specific antibodies, anti-MSP1-19, anti-DBP and anti-Pvs16, the antibody-based FC can be used to monitor the maturation of $P$. vivax parasite in the culture. The antibody based-FC using anti-PfBiP is a very sensitive and highly accurate method, which offers the new way to detect parasite in vivax malaria research.

Table 2 Differential staining pattern of $P$. vivax

\begin{tabular}{lllll}
\hline Stage & BiP & MSP1 & DBP & PVS16 \\
\hline Ring to Mid-stage Trophozoite & + & - & - & - \\
Late-Trophozoite-early schizont & + & + & - & - \\
Mid-late Schizont & + & + & + & - \\
Gametocyte & + & - & - & + \\
\hline
\end{tabular}

Ring to mid trophozoite were stained with BiP, late trophozoite to early schizont were stained with BiP and MSP1, mid to late schizont were stained with BiP, MSP1 and DBP, and gametocyte was stained with BiP and pvs16.

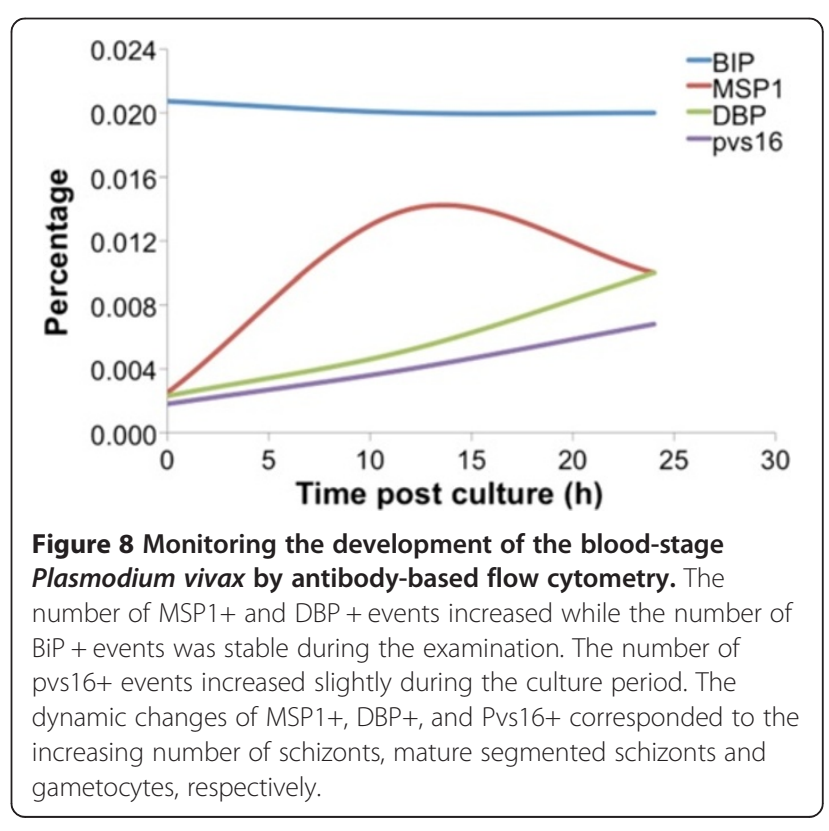




\section{Abbreviations}

BiP: Binding immunoglobulin protein of endoplasmic reticulum; DBP: Duffy binding protein; FC: Flow cytometry; IFA: Immunofluorescence assay; PVMSP1: Plasmodium vivax merozoite surface protein-1; PBS: Phosphatebuffered saline; PBST: PBS containing Tween-20; PfBiP: Plasmodium falciparum BiP; Pvs16: Plasmodium vivax sexual antigen 16; WBC: White blood cells.

\section{Competing interests}

The authors declare that they have no competing interests.

\section{Authors' contributions}

WR and JHA conceived of the study. WR, SPM, JS and JHA designed the experiments. WR, SPM, NR, and SJB carried out the experiments. WR, SJB and $J H A$ drafted the manuscript. All authors read and approved the final manuscript.

\section{Acknowledgements}

This work was supported by grants NIH R01Al064478 (JHA), NIH R01Al069314 (KCW), and Bill \& Melinda Gates Foundation (JHA). We thank the P. vivax patients and residents of Kanchanaburi Province, Thailand for their generosity for participation in the study.

\section{Author details}

'Department of Global Health, College of Public Health, University of South Florida, Tampa, FL, USA. ${ }^{2}$ Mahidol Vivax Research Unit, Faculty of Tropical Medicine, Mahidol University, Bangkok, Thailand. ${ }^{3}$ Department of Biology, Loyola University, Chicago, IL 60660, USA.

Received: 2 October 2013 Accepted: 22 January 2014

Published: 14 February 2014

\section{References}

1. Guerra CA, Howes RE, Patil AP, Gething PW, Van Boeckel TP, Temperley WH, Kabaria CW, Tatem AJ, Manh BH, Elyazar IR, Baird JK, Snow RW, Hay Sl: The international limits and population at risk of Plasmodium vivax transmission in 2009. PLoS Negl Trop Dis 2010, 4:e774.

2. Kitchen SK: The infection of reticulocytes by Plasmodium vivax. Am J Trop Med Hyg 1938, 18:347-353.

3. Malleret B, Xu F, Mohandas N, Suwanarusk R, Chu C, Leite JA, Low K, Turner C, Sriprawat K, Zhang R, Bertrand O, Colin Y, Costa FT, Ong CN, Ng ML, Lim CT, Nosten F, Rénia L, Russell B: Significant biochemical, biophysical and metabolic diversity in circulating human cord blood reticulocytes. PLoS One 2013, 8:e76062

4. Grimberg BT, Erickson JJ, Sramkoski RM, Jacobberger JW, Zimmerman PA: Monitoring Plasmodium falciparum growth and development by UV flow cytometry using an optimized Hoechst-thiazole orange staining strategy. Cytometry A 2008, 73:546-554.

5. Hare JD, Bahler DW: Analysis of Plasmodium falciparum growth in culture using acridine orange and flow cytometry. J Histochem Cytochem 1986, 34:215-220.

6. Kosaisavee V, Suwanarusk R, Nosten F, Kyle DE, Barrends M, Jones J, Price R, Russell B, Lek-Uthai U: Plasmodium vivax: isotopic, PicoGreen, and microscopic assays for measuring chloroquine sensitivity in fresh and cryopreserved isolates. Exp Parasitol 2006, 114:34-39.

7. Pattanapanyasat $\mathrm{K}$, Yongvanitchit $\mathrm{K}$, Tongtawe $\mathrm{P}$, Tachavanich $\mathrm{K}$, Wanachiwanawin W, Fucharoen S, Walsh DS: Impairment of Plasmodium falciparum growth in thalassemic red blood cells: further evidence by using biotin labeling and flow cytometry. Blood 1999, 93:3116-3119.

8. Staalsoe T, Giha HA, Dodoo D, Theander TG, Hviid L: Detection of antibodies to variant antigens on Plasmodium falciparum-infected erythrocytes by flow cytometry. Cytometry 1999, 35:329-336.

9. Theron M, Hesketh R, Subramanian S, Rayner J: An adaptable two-color flow cytometric assay to quantitate the invasion of erythrocytes by Plasmodium falciparum parasites. Cytometry A 2010, 77:1067-1074.

10. Kumar N, Syin C, Carter R, Quakyi I, Miller LH: Plasmodium falciparum gene encoding a protein similar to the $78-\mathrm{kDa}$ rat glucose-regulated stress protein. Proc Natl Acad Sci U S A 1988, 85:6277-6281.

11. Noe AR, Fishkind DJ, Adams JH: Spatial and temporal dynamics of the secretory pathway during differentiation of the Plasmodium yoelii schizont. Mol Biochem Parasitol 2000, 108:169-185.
12. Hager KM, Striepen B, Tilney LG, Roos DS: The nuclear envelope serves as an intermediary between the ER and golgi complex in the intracellular parasite Toxoplasma gondii. J Cell Sci 1999, 112:2631-2638.

13. Russo I, Oksman A, Vaupel B, Goldberg DE: A calpain unique to alveolates is essential in Plasmodium falciparum and its knockdown reveals an involvement in pre-S-phase development. Proc Natl Acad Sci U S A 2009, 106:1554-1559.

14. van Dooren GG, Marti M, Tonkin CJ, Stimmler LM, Cowman AF, McFadden GI: Development of the endoplasmic reticulum, mitochondrion and apicoplast during the asexual life cycle of Plasmodium falciparum. Mol Microbiol 2005, 57:405-419.

15. Janse CJ, Camargo A, Delportillo HA, Herrera S, Waters AP, Kumlien S, Mons B, Thomas A: Removal of leucocytes from Plasmodium vivax-infected blood. Ann Trop Med Parasit 1994, 88:213-216

16. Udomsangpetch R, Somsri S, Panichakul T, Chotivanich K, Sirichaisinthop J Yang Z, Cui L, Sattabongkot J: Short-term in vitro culture of field isolates of Plasmodium vivax using umbilical cord blood. Parasitol Int 2007, 56:65-69.

17. Ntumngia FB, Schloegel J, Barnes SJ, McHenry AM, Singh S, King CL, Adams $\mathrm{JH}$ : Conserved and variant epitopes of Plasmodium vivax Duffy binding protein as targets of inhibitory monoclonal antibodies. Infect Immun 2012, 80:1203-1208.

18. Fennell C, Babbitt S, Russo I, Wilkes J, Ranford-Cartwright L, Goldberg DE, Doerig C: PfelK1, a eukaryotic initiation factor 2alpha kinase of the human malaria parasite Plasmodium falciparum, regulates stress-response to amino-acid starvation. Malar J 2009, 8:99.

19. Kumar N, Koski G, Harada M, Aikawa M, Zheng H: Induction and localization of Plasmodium falciparum stress proteins related to the heat shock protein 70 family. Mol Biochem Parasitol 1991, 48:47-58.

20. Aurrecoechea C, Brestelli J, Brunk BP, Dommer J, Fischer S, Gajria B, Gao X, Gingle A, Grant G, Harb OS, Heiges M, Innamorato F, lodice J, Kissinger JC, Kraemer E, Li W, Miller JA, Nayak V, Pennington C, Pinney DF, Roos DS, Ross C, Stoeckert CJ Jr, Treatman C, Wang H: PlasmoDB: a functional genomic database for malaria parasites. Nucleic Acids Res 2009, 37:D539-D543

21. Klemba M, Gluzman I, Goldberg DE: A Plasmodium falciparum dipeptidyl aminopeptidase I participates in vacuolar hemoglobin degradation. J Biol Chem 2004, 279:43000-43007.

22. Kumar N, Nagasawa H, Sacci JB, Sina BJ, Aikawa M, Atkinson C, Uparanukraw P, Kubiak LB, Azad AF, Hollingdale MR: Expression of members of the heat-shock protein-70 family in the exoerythrocytic stages of Plasmodium-berghei and Plasmodium-falciparum. Parasitol Res 1993, 79:109-113.

23. Apte SH, Groves PL, Roddick JS, PdH V, Doolan DL: High-throughput multi-parameter flow-cytometric analysis from micro-quantities of Plasmodium-infected blood. Int J Parasitol 2011, 41:1285-1294.

24. Fu Y, Tilley L, Kenny S, Klonis N: Dual labeling with a far red probe permits analysis of growth and oxidative stress in $P$. falciparum-infected erythrocytes. Cytometry A 2010, 77:253-263.

25. Russell B, Suwanarusk R, Borlon C, Costa FT, Chu CS, Rijken MJ, Sriprawat K, Warter L, Koh EG, Malleret B, Colin Y, Bertrand O, Adams JH, D'Alessandro U, Snounou G, Nosten F, Rénia L: A reliable ex vivo invasion assay of human reticulocytes by Plasmodium vivax. Blood 2011, 118:e74-e81.

\section{doi:10.1186/1475-2875-13-55}

Cite this article as: Roobsoong et al:: A rapid sensitive, flow cytometry-based method for the detection of Plasmodium vivax-infected blood cells. Malaria Journal 2014 13:55. 